\title{
Acrylamide content in French fries prepared in food service establishments
}

\author{
Marta Mesías, Cristina Delgado-Andrade, Francisca Holgado, Francisco J. Morales
}

Institute of Food Science, Technology and Nutrition, ICTAN-CSIC, Madrid 28040, Spain

\begin{abstract}
Since the regulation on the acrylamide reduction in foods, the food business operators have implemented mitigation strategies for reduction of acrylamide content in French fries. Thirty food service establishments from ten of the most relevant trademark food restaurants were recruited. Par-fried frozen potato, fried potato, and frying oil were collected at two different moments of the day (lunch/dinner) for two different days. Acrylamide, reducing sugar content, moisture, colour, and polar compounds were measured. Acrylamide content $(<20-1068 \mu \mathrm{g} / \mathrm{kg})$ and the mean $(303 \mu \mathrm{g} / \mathrm{kg})$ were in line with the EFSA estimations for French fries. 13.5\% of the samples reported acrylamide above $500 \mu \mathrm{g} / \mathrm{kg}$ (benchmark level settled for the EU Regulation 2158/2017). Regardless frying conditions applied and operational procedures, the reducing sugar content $(<0.2-4.89 \mathrm{~g} / \mathrm{kg})$ in the unprocessed potato, the colour parameter $\mathrm{a}^{*}(-2.56-5.2 \mathrm{l})$, and the moisture content (29.26-65.93\%) of the French fries correlated significantly to acrylamide. The colour parameter $\mathrm{a}^{*}$ is useful to set up chemometric tools to provide rapid and cost-effective control for acrylamide content. Increasing the level of automatization will reduce the acrylamide variability among food service establishments and will minimize the impact of inadequate decisions of the food handler.
\end{abstract}

KEYWORDS: Acrylamide, French fries, Food services, Frying, Colour.

\section{INTRODUCTION}

The European Food Safety Authority (EFSA) concluded that levels of dietary exposure to acrylamide across age groups indicate a concern due to its carcinogenic effects (EFSA, 2015). European Commission stablished in 2017 mitigation measures for the reduction of acrylamide in foods and identified new benchmark levels for a number of food categories (European Commission, 2017). The Regulation 2158/2017 compels food processors and food business operators in Europe to reduce the presence of acrylamide in their products according to the ALARA principle, establishing measures proportionate to the size and nature of the establishments. Potato-based products are one of the main contributors to acrylamide exposure (EFSA, 2015), and a benchmark level of $500 \mu \mathrm{g} / \mathrm{kg}$ has been settled for French fries ready-to-eat (European Commission, 2017).

French fries are prone to form acrylamide due to the high content of precursors in the tuber and the intensity of the thermal treatment applied. Chemical composition of potato varies with cultivar, location of growth, agricultural practices, maturity at harvest, storage history, among others (Medeiros Vinci et al., 20II). Deep-fat frying is one of the oldest and most common unit operation used for cooking foods by immersing them in an edible oil or fat heated above the boiling point of water (Farkas, Singh, \& Rumsey, 1996). Frying generates a composite structure formed by an external dehydrated, porous and crispy outer layer or crust region where oil is located and a moist and cooked interior or core region free of oil (Pedreschi, 2009; Pedreschi \& Moyano, 2005). The majority of the acrylamide forms at the surface and in nearsurface regions of the French fries where the highest temperatures are attained (Palazoglu \& Gökmen, 2008). However, these former investigations have been carried out in the laboratory, under controlled conditions and conclusions can not be directly extrapolated to the food service establishment. 
French fries are commonly prepared in food service establishments. The EU Regulation stated that the levels of acrylamide can be lowered by mitigation approaches, such as implementation of good manipulation practices and application of procedures based on hazard analysis and critical control point principles (European Commission, 2017). Mitigation strategies in potato products involve the selection of varieties with low sugar content, adequate storage and transport, suppress sprouting, blanching, disodium diphosphate addition, asparaginase treatment, cut thicker, frying at maximum $175^{\circ} \mathrm{C}$, among others (FoodDrinkEurope, 2013). However, their application on the industrial production of French fries do not always result in acrylamide mitigation (Medeiros Vinci et al., 201I). The implementation at the domestic setting, catering services or restaurants has been poorly addressed, or even lacking. Sanny, Luning, Marcelis, Jinap, and Van Boekel $(2010,2013)$ proposed that food handler's inadequate control leads to high variations in acrylamide. Recently, home cooking practices for the preparation of French fries from fresh potatoes in Spanish homes reported levels of acrylamide 2-fold higher than the average estimation of the EFSA report in this food category (Mesias, Delgado-Andrade, Holgado, \& Morales, 2018). The EFSA' experts opinion cited that occurrence of acrylamide in a domestic setting could be increased up to $80 \%$ (EFSA, 20I5). Then, frying practices of households and public food establishments have not properly considered in the estimation of the acrylamide exposure risk.

Eating out of home is growing and has gained an important place in the habitual diet for Western countries and, preferably in the urban environments (Lachat et al., 2012). The food operators from canteens and restaurants have become a strategic stakeholder to promote a balanced diet among citizens, but also may reduce the exposure to acrylamide across Europe. However, mitigation practices are not extensively implemented to the restaurants. This investigation aimed to evaluate acrylamide levels in French fries prepared in food service establishments that operate under one trademark or commercial license. The relationship between the variables taking part during the preparation of French fries has been evaluated in order to identify critical points for the mitigation of acrylamide.

\section{MATERIALS AND METHODS}

\section{Chemicals and materials}

Formic acid (98\%), D(+)-Glucose and methanol (99.5\%) were from Panreac (Barcelona, Spain). I3C3-labelled acrylamide ( $99 \%$ isotopic purity) was obtained from Cambridge Isotope Laboratories (Andover, MA, USA). Deionized water was obtained from a Milli-Q Integral 5 water purification system (Millipore, Billerica, MA, USA). All other chemicals, solvents and reagents of analytical grade were obtained from Sigma (St. Louis, USA).

\section{Study design}

Ten brands of chain restaurants (RI, R2, R3, R4, R5, R6, R7, R8, R9, and RI0) operating under a trademark were recruited. The Supplementary material I depicts the experimental design. Each restaurant participated with three different food service establishments placed in the province of Madrid (Spain). Sampling at each establishment $(n=30)$ was scheduled in two different days (day-I, day-2), within a period of at least 3 weeks. This interval for sampling ensured the use of different lots of par-fried frozen potatoes. In this research, for simplification in the sample description, the term 'unprocessed sample' refers to the sample before deepfrying, regardless of the pre-treatment applied. Two different moments in the same day were selected for sampling (lunch, dinner) to evaluate the contribution of the food handlers. Restaurants did not receive instructions for the potato variety or supplier, manipulation, fryer, frying temperature or frying oil. The food handlers used the potato supplier, frying appliance, frying oil, serving size, potato/oil ratio, and end-point following the standardised protocol for frying of French fries stablished by the restaurant in order to reflect 
the common practices. In this way, a randomised scenario of the food establishment practices for the preparation of French fries in different chain restaurants was covered. Restaurant' managers are interviewed about characteristics of the suppliers for frozen potato and the frying operations. Due to the confidentiality agreement signed with them some details of the internal operating procedures for frying (initial and end frying temperature and frying time, potato-to-oil ratio, and details of the fryer equipment) were not provided. The study was limited to par-fried frozen strips intended for the preparation of French fries. The food handlers were unaware of the purpose of the research and not informed in detail about the acrylamide issue in order to avoid any influence during the experiment. The study was conducted from April to May 2017.

\section{Sampling}

Restaurants received a 'kit of sampling' containing a guidance with instructions for sampling according to the study design and containers properly coded to dispatch the samples. Each establishment collected a batch of par-fried potato strips (named as unprocessed sample), a batch of French fries (fried sample) and an aliquot (ca. $25 \mathrm{~mL}$ ) of frying oil. Frying oil was collected just after the frying of the sample. There was not a specific instruction to collect the sample after a certain number of cycles of frying. Once cooled, the fried potato and the frying oil were kept frozen together with the unprocessed potatoes. Then, each restaurant collected 12 samples of unprocessed potato strips, 12 samples of French fries and 12 samples of frying oil at two moments of the day, for two different days at three different establishments. R2 only provided three groups of samples (unprocessed potato, fried potato, frying oil) corresponding to the first day of sampling at lunch time, and then the total number of batches of samples collected was III, instead of 120. Frozen samples were placed in an airtight freezer bag and couriered to the lab. After reception, samples were stored at $-20{ }^{\circ} \mathrm{C}$ until analysis.

\section{Determination of moisture content}

Moisture was determined gravimetrically according to the Association of Official Analytical Chemists (AOAC) method 950.46 (AOAC, 1995).

\section{Determination of reducing sugar content}

The determination of the reducing sugar (glucose + fructose) content in unprocessed potato strips was as described by Miller (1959) with the modifications from Mesías, Holgado, Márquez-Ruiz, and Morales (2017) as adapted to a plate-reader. Results were expressed as g glucose equivalents $/ \mathrm{kg}$ of fresh sample. Limit of quantification (LoQ) was set at $0.2 \mathrm{~g}$ glucose equivalents $/ \mathrm{kg}$ sample.

\section{Determination of colour}

Colour measurements were made at room temperature using a HunterLab Spectrophotometer CM-3500D colorimeter (Hunter Associates laboratory, Stamford, Connecticut, USA). Four independent measurements of $a^{*}$ (redness), $b^{*}$ (yellowness) and $L^{*}$ (lightness) parameters were carried out on different areas of fried potatoes in order to take into account the non-homogeneous distribution of the colour within the same batch of fried product. The sample is placed in a $34 \mathrm{~mm}$ optical glass cell, and illuminated with a D65-artificial daylight $\left(10^{\circ}\right.$ standard angle). $E$ index was calculated according to the following equation: $E=\left(L^{2}+a^{2}+b^{2}\right)^{1 / 2}$. The equipment was calibrated with a standard calibration white plate CR-A43 (L*/93.80, a*/0.3I56, b*/ $0.3319)$.

Acrylamide determination by liquid chromatography-electrospray ionization-tandem mass spectrometry 
Acrylamide was determined in fried samples as described by Mesías and Morales (2015). The recovery of the procedure was between $90 \%$ and $106 \%$. The relative standard deviations for precision, repeatability and reproducibility were $2.8 \%, 1.2 \%$ and $2.5 \%$, respectively. The limit of detection is set at $20 \mu \mathrm{g} / \mathrm{kg}$ for potatobased foods. The accuracy of the results were recently demonstrated for potato-based matrix (potato crisps) in three proficiency tests launched by the Food Analysis Performance Assessment Scheme (FAPAS®) program, yielding a z-score of 0.2 (Test 3065, May-June 2016), - 0.2 (Test 307I, Feb-March 2017) and - 0.3 (Test 3080, Feb-March 2018). Analysis was performed by duplicate and results were expressed as $\mu \mathrm{g} / \mathrm{kg}$ of sample.

\section{Determination of polar compounds in frying oil}

Total polar compounds in the frying oil were measured with a Testo 270 (Testo INC, New Jersey, USA). Results were expressed as percentage of total polar material (g/l00 g oil).

\section{Statistical analysis}

Statistical analyses were performed by using SPSS version 22.0 (SPSS Inc., Chicago, IL). Analysis were done at least by duplicate. Data are expressed as fresh weight. The statistical significance of the variables were tested applying analysis of variance (ANOVA one-way) and the test of mean comparison according to Fisher least significant difference (LSD). Homogeneity of variances was determined with the Levene's test. Relationships between the different variables were evaluated by computing Spearman's linear correlation coefficient (Petrie \& Sabin, 2009). All statistical parameters were evaluated at $p<0.05$ significance level.

\section{RESULTS AND DISCUSSION}

\section{Description of the restaurants and protocols of frying French fries}

Table I summarises the characteristics of the unprocessed (par-fried frozen) potato and frying operations of the restaurants. All the restaurants used commercial par-fried frozen potatoes intended for frying from the major distributors across Europe. Suppliers for frozen potato strips provide cooking instructions on the label of every pack (fry at maximum $175^{\circ} \mathrm{C}$ for a light golden colour, and do not overcook). During the production of par-fried potato, the peeled potatoes are cut along the long axis to produce strips with predetermined cross sectional areas that vary depending on the processor or the consumer preferences. Most of the samples were classic straight cut frozen French fries, except for R2 and R6 which were rustic fried potatoes 'skin on' and rustic chips 'medallion', respectively. None of the restaurants thawed the potatoes before frying. The reducing sugar content in the unprocessed potato used by the restaurants was not controlled by them, nor described by the potato supplier.

Regarding to the frying conditions, all the restaurants used high oleic sunflower oil as frying oil. Five restaurants stablished a reference frying temperature slightly above $175^{\circ} \mathrm{C}$. Electric fryer and gas fryers with a vessel of at least II L of capacity were commonly used. However, there were important differences in the frying technology among restaurants, and even among the establishments for the same restaurant. There were two types of fryers according to the temperature control, a manual dial thermostat controller or a computer process controller. These controllers automatically detect the load size, bring temperatures up quickly and precisely and then lower the temperature or extent/reduce the cooking time to complete the frying. In most of the restaurant, there is not a dedicated personnel exclusively assigned to a deep-frying station. All the restaurants have internal operational guidelines for frying in batch.

\section{Characterization of the unprocessed potatoes, fried potatoes and frying oil}


Unprocessed potato, fried potato, and frying oil samples were characterized for the content of precursors and parameters that may play an important role in the acrylamide formation. Table 2 summarises the reducing sugar content in the unprocessed potato strips, moisture in both unprocessed and fried potatoes, colour (CIELab) in French fries and polar compounds in frying oil in the batches of samples provided by the restaurants.

The reducing sugar content in the unprocessed potato strips $(n=\mathrm{III})$ ranged from $<\mathrm{LOQ}(0.2 \mathrm{~g} / \mathrm{kg})$ to 4.89 $\mathrm{g} / \mathrm{kg}$. The mean value was $1.3 \mathrm{I} \mathrm{g} / \mathrm{kg}$ and the median was set at $1.37 \mathrm{~g} / \mathrm{kg}$. It is noteworthy to mention that $49.6 \%$ of the samples $(n=55)$ reported a reducing sugar content lower than LOQ. Results were expected, since the procedure for the elaboration of par-fried frozen potato strips intended for frying includes practices that reduce the levels of reducing sugars, i.e. blanching (Pedreschi, 2009). Moisture content in the unprocessed potatoes ranged from 61.43 to $75.67 \%$, with a mean of $66.98 \%$ and a median of $66.67 \%$. Moisture content needs to be less than $70 \%$ in par-fried potatoes to prevent limpness and separation of the interior and the crust (Bradshaw \& Ramsay, 2017). The average and median moisture content in French fries were $45.83 \%$ and $45.02 \%$, respectively. Results are in line with those reported in the literature that are up to $38 \%$ of moisture content the final product (Saguy \& Dana, 2003). French fries show large differences in the colour parameters among restaurants, varying from light colour (minimum $L^{*}$ of 70.23 ) to darker colour (maximum $L^{*}$ of 53.32). However, most of the restaurants adjusted the frying protocol to reach an adequate homogeneous golden colour (median $L^{*}$ of 63.89 ). The quality of the frying oil after a number of frying cycles is determined by the percentage of total polar materials, setting the limit in $25 \%$ (Dobarganes \& MárquezRuiz, 1998). The daily determination of polar compounds is implemented in the frying protocol of the restaurants. The total polar compounds ranged from 3.30 to $36.80 \mathrm{~g} / 100 \mathrm{~g}$ oil. The mean value was 10.44 $\mathrm{g} / 100 \mathrm{~g}$ oil and the median was set at $8.50 \mathrm{~g} / 100 \mathrm{~g}$ oil. There were six samples with values higher than 25 $\mathrm{g} / \mathrm{l} 00 \mathrm{~g}$ oil, which represents $5.4 \%$ of the sampling. Four of these samples come from the same food service establishment of the R3 that denotes a fail in the supervision of the process.

\section{Acrylamide levels in French fries prepared in food establishments}

The acrylamide content showed a high variability, with values ranging from<LOQ $(20 \mu g / \mathrm{kg})$ to $1068 \mu \mathrm{g} / \mathrm{kg}$ (Table 2). The mean was $303 \mu \mathrm{g} / \mathrm{kg}$ and the median was set at $265 \mu \mathrm{g} / \mathrm{kg}$. These values are in line with the outcome of the EFSA scientific opinion on acrylamide in fried potato products that determines an average content of $308 \mu \mathrm{g} / \mathrm{kg}$ (EFSA, 20I5). One sample contained levels of acrylamide exceeding $1000 \mu \mathrm{g} / \mathrm{kg}$, and two samples exhibited an acrylamide content lower than the LOQ. Recently, the European Commission has established new benchmark levels for the main foodstuffs contributors to the acrylamide exposure, 500 $\mu \mathrm{g} / \mathrm{kg}$ being the reference settled for French fries (European Commission, 2017). According to these recommendations, the proportion of samples containing acrylamide at a level above $500 \mu \mathrm{g} / \mathrm{kg}$ was $13.5 \%$ $(n=15)$ (Fig. I).

The distribution of the acrylamide content within restaurants was studied more in detail to identify potential sources of variability that could explain the acrylamide variation (Fig. 2A). The mean acrylamide content was I55, I67, 25I, 256, 277, 282, 297, 39I, 420, and $604 \mu \mathrm{g} / \mathrm{kg}$ for restaurants R4, R7, R5, R8, RI0, R6, R3, R2, RI, and R9, respectively. The type of cut (rustic chips and classic French fries) had no influence. Five restaurants reported samples with acrylamide above the benchmark value, although 9 of the 15 samples were from the same restaurant (R9). R4 reported two samples with values < LOQ. There were significant differences among restaurants for acrylamide in French fries (Supplementary material 2A). 
It is noteworthy to mention that this study covers three different establishments for each restaurant, including two days for each establishment and two moments (lunch and dinner) for each day that increases greatly the variability. However, most of the restaurants obtained a good reproducibility for acrylamide, being lower than $50 \%$ (from $21.4 \%$ to $47.6 \%$ ). R9 and R2 exceeded this value, showing $56 \%$ and $64 \%$ of variability, respectively (Fig. 2A). These restaurants had not a precise control of the frying conditions, or an inadequate supervision by the food operator. In this sense, a major adjustment in the process together with a better training of the operator could be applied to reduce the acrylamide content in their French fries. Results for $\mathrm{RI}$ are also interesting to discuss since this restaurant exhibited the best reproducibility (21.4\%) among all the three establishments with mean values of $403 \pm 41.2,426 \pm 113.8$, and $430 \pm 119.8 \mu \mathrm{g} / \mathrm{kg}$ of acrylamide for each independent establishment (Fig. 2A). RI performs a frying process with a high level of standardization and supervision. In contrast, the frying process was uncontrolled or not reproducible for R9, where French fries presented acrylamide concentrations ranging from 65 to $1068 \mu \mathrm{g} / \mathrm{kg}$. In agreement with Sanny et al. (2013), these results pointed out that appropriate operational instructions to handlers will reinforce an effective decision-making process in out of control situations to result in minimize the variation in acrylamide.

The variations in acrylamide within restaurants is a consequence of the frying conditions, the implication of human behaviour to set the end of frying, but also due to the characteristics of the potatoes intended for frying. The content of reducing sugars in par-fried frozen potatoes has been directly correlated with the formation of acrylamide for French fries (Fiselier \& Grob, 2005). The reducing sugar content of the frozen potatoes is depicted in Fig. 2B. The median reducing sugar content $(\mathrm{g} / \mathrm{kg})$ was $<\mathrm{LOQ}(\mathrm{R} 2, R 6, R 8, R 5), 0.84$ (RI0), 0.94 (R7), 1.04 (R4), 2.19 (R3), 2.35 (RI), and 2.27 (R9). ANOVA one-way analysis was applied for identifying differences in the means (Supplementary material $2 \mathrm{~B}$ ). Considering all the restaurants together, a significant relationship was found between the reducing sugar content in the unprocessed potatoes (par-fried frozen) and the acrylamide content in the French fries $(\rho=0.3586, p=0.0003)$ (Table 3). The Pearson's correlation coefficients were slightly strongest if restricted for classical straight cut frozen potatoes. Taubert, Harlfinger, Henkes, Berkels, and Schömig (2004) found that in potato shapes with a low surface over volume ratio, acrylamide consistently increased while temperature and processing time increased as well. $\mathrm{RI}$ and $\mathrm{R} 9$ showed the highest reducing sugar content (RI: $2.31, \mathrm{R} 9: 2.59 \mathrm{~g} / \mathrm{kg}$ ) and the highest acrylamide content (RI: $420 \mu \mathrm{g} / \mathrm{kg}, \mathrm{R} 9: 604 \mu \mathrm{g} / \mathrm{kg}$ ). Similarly, R6, R8 and R I0, with low levels of acrylamide, used unprocessed potato strips with the lowest reducing sugar content, most of the samples presenting values under the LOQ. R2 is an exception in this trend since reported the lowest reducing sugar content $(<L O Q)$, but obtained an unexpected relatively high acrylamide content $(391 \pm 250 \mu \mathrm{g} / \mathrm{kg})$. The acrylamide toolbox recommends a reducing sugar content lower than $3 \mathrm{~g} / \mathrm{kg}$ for fresh potatoes intended for French fries preparation. A limit for reducing sugars at $0.7 \mathrm{~g} / \mathrm{kg}$ to reduce the exposure to acrylamide is also recommended (Fiselier \& Grob, 2005). In the present study, 9 samples showed values higher than $3 \mathrm{~g} / \mathrm{kg}$, and 47 samples (42\%) reported values between 0.7 and $3 \mathrm{~g} / \mathrm{kg}$.

\section{Relationship with reducing sugars, moisture and colour}

Fig. $3 \mathrm{~A}$ depicts the distribution of the average acrylamide content in the fried potatoes and the reducing sugar content in the unprocessed potatoes for each restaurant. Black circles denote restaurants with rustic fried potatoes and white circles denote restaurants with classic French fries straight cut. It is observed that the critical level of reducing sugar content for the preparation of French fries is set to $2.5 \mathrm{~g} / \mathrm{kg}$, regardless the type of cut or the frying procedure applied. Higher sugar content involves higher acrylamide formation, exceeding then the benchmark levels. Acrylamide reduction is obtained using low reducing sugar potato varieties and processes such as blanching for leaching the excess of reducing sugars (Medeiros Vinci et al., 
20II). Unfortunately, the restaurants were not able to report specific information on the supplier of parfried frozen potato strips. These results confirm in a real scenario for food service establishments the relevance of using par-fried frozen potatoes with a low content in reducing sugars as a critical strategy for the acrylamide mitigation.

Moisture is related to the acrylamide content (Table 3). Taking all the restaurants together, significant relationships with acrylamide content were found for the moisture content in the par-fried frozen potatoes $(\rho=0.2238, p=0.0284)$, in the French fries $(=0.3832, p=0.000 \mathrm{I})$, and the moisture loss $(\rho=0.3058, p=0.0024)$. The moisture of frozen par-fried potatoes for the different establishments of the same restaurant showed a high reproducibility, which suggests the same potato supplier. The loss of moisture ranged from $1.95 \%$ to the $38.18 \%$, with an average of $21.14 \%$ and a median of $22.28 \%$. The lowest moisture loss was for R6 (I3.I I $\pm 4.52 \%)$, and the highest lost for R9 $(27.75 \pm 3.91 \%)$, and R2 $(23.76 \pm 7.93 \%)$ that corresponds with the levels of acrylamide. However, the moisture in fried products gave the strongest correlation with acrylamide, regardless the restaurant. Fig. 3B depicts the distribution of the average acrylamide content and the moisture in the fried potatoes for each restaurant. A frying process that reduces the moisture content below $40 \%$ will likely reach an acrylamide content higher than the benchmark level in a real scenario for food service establishments.

Colour is one of the critical parameters to decide the end-point for frying. Colour in the fried potato is the result of the extent of the Maillard reaction that depends on the superficial reducing sugar content, temperature and frying period, among other variables like moisture content (Pedreschi, Kaack, \& Granby, 2006). The CIELab colour parameters were evaluated in the French fries (Table 2). A significant correlation was found between acrylamide and parameter $L^{*}(\rho=-0.2117, p=0.0384)$. A stronger relationship was found with the colour parameter $a^{*}(\rho=0.7172, p<0.0000)$ (Table 3$)$. A linear correlation between acrylamide content of potato chips and the chromatic redness component $\mathrm{a}^{*}$ has been previously reported for laboratory experiments under controlled conditions (Pedreschi \& Moyano, 2005). Fig. 4 depicts the linear correlation between acrylamide and colour parameter $a^{*}$ in French fries straight cut. Since the geometrical dimensions of the pieces may influence the acrylamide formation, R2 and R6 were skipped from the dataset to avoid bias related to the type of French fries, and only classical French fries straight cut $(n=96)$ were depicted. Three samples were outside the interval of confidence for the linear regression that corresponds to R9. This restaurant reported some samples with a content in polar compounds higher than $25 \%$ that denotes an extensive use of the frying oil. It could be possible that colour of those samples that did not follow the general trend was influenced by the darkening of the frying oil. Results confirms that colour $\mathrm{a}^{*}$ parameter is a reliable and sensible variable to assess the acrylamide content in French fries ready-to-eat as prepared in food service establishments. These results support the application of chemometric devices based in colour parameters in food service establishments for monitoring acrylamide levels as proposed in the Regulation for acrylamide.

\section{Relationship with the frying process and frying oil}

The characteristic of the fryer and frying process for each restaurant is described in Table I. Most of the information concerning the fryer and the frying parameters were not able to be published in detail. Restaurants used both electric and gas conventional fryers in batch. There is not information if food service establishments only used atmospheric fryers. Few restaurants reported information about the temperature/time cycle and potato-to-oil ratio in the basket. The extent of treatment is settled for each restaurant according to its operational practices, convenience and desirable sensory properties expected by customers. Some of the restaurants used a preselected temperature based on the instruction of the potato 
supplier, the fryer equipment or based on their own experience. However, as described by Fiselier, Hartmann, Fiscalini, and Grob (2005), the critical point of the frying process is not only represented by the initial oil temperature but also by the real temperature of the oil and potato surface during the frying process. In addition, the decreasing of initial oil temperature after the immersion of potato sticks and the duration of the process are strictly dependent on the product-to-oil ratio and on the heating power of the fryer.

All the restaurants used high-oleic sunflower oil (Table I). To date there is still some controversial regarding the influence of the oxidative and hydrolytic degradation of the frying oil on acrylamide formation. Lipid oil oxidation may rise the level of carbonyl compounds, which are the precursors of acrylamide, (Zamora \& Hidalgo, 2008), but net increases of acrylamide have been stated only in lipid-fat foods and particularly in dry-foods (Capuano, Oliviero, Açar, Gökmen, \& Fogliano, 2010). It has been hypothesized that the acrylamide formation pathway from lipids may exist, since triacylglycerols partially hydrolyze during frying, followed by dehydration of glycerol to acrolein that may oxidize to acrylic acid, which can finally react with ammonia to form acrylamide (Gertz \& Klostermann, 2002). However, Mestdagh, Castelein, Van Peteghem, and De Meulenaer (2008) concluded that the contribution of acrolein and other oil degradation products to the overall formation of acrylamide in fried stuffs is neglible. Additionally, the acrylamide formation in French fries was independent upon oil oxidation and hydrolysis status (Mestdagh, De Meulenaer, \& Van Peteghem, 2007). A recent investigation stated that a higher percentage of unsaturated fatty acids in the oil composition did not cause an increase in the acrylamide content in French fries regardless the number of frying cycles (Zhang, Zhang, Cheng, Wang, \& Qian, 2015). In the present study, acrylamide levels in French fries were not significantly correlated with polar compounds in frying oil $(p>0.05)$ (Table 3$)$.

Frying is extensively used in food service establishments and in the domestic setting, due to its ability to create unique sensory properties, including texture, flavour and appearance, which makes the food more palatable and desirable (Saguy and Dana, 2003). There are different strategies of mitigation that could be implemented at food establishments. Palermo et al. (2016) identified three key parameters to be considered before the introduction of a mitigation strategy for acrylamide: (i) the reduction rate obtained, (ii) evaluate potential side effects, and (iii) the applicability and economic impact of its implementation. These decisions will influence the variation of the acrylamide concentration below the benchmark level. The present study confirms that controlling the reducing sugar content is the simplest and most effective mitigation strategy for food service establishments. A precise control of the supplier for par-fried frozen potatoes in terms of reducing sugar level in the product reveals as critical.

\section{CONCLUSIONS}

The food business operators in the Spanish restaurant sector currently compiles with the benchmark level for French fries ready-to-eat as described in the Regulation for acrylamide. However, it is necessary to continue working in reducing the acrylamide levels in French fries since the benchmark levels will be revised every three years and a reduction in them will likely occur. The content of reducing sugar in the par-fried frozen potatoes intended for frying is a critical parameter that should be as low as agronomical and/or technologically possible. Food business operators should stablish in their operational protocols a maximum reducing sugar content of $2.5 \mathrm{~g} / \mathrm{kg}$ in par-fried frozen potato intended for frying. Therefore, food establishments should reinforce the selection of par-fried frozen potatoes with a low content in reducing sugars with the aim of contributing to the reduction of acrylamide exposure to population. Additionally, the chromatic parameter $a^{*}$ is an useful reference to set up chemometric tools in order to provide rapid and cost-effective control for acrylamide content in French fries served in food establishments. Increasing the 
degree of automatization and control of the frying process will reduce the acrylamide variability among establishments for the same restaurant and will reduce the impact of any inadequate decision of the food handler.

\section{DECLARATION OF INTEREST}

The authors declare that they have no conflict of interest.

\section{ACKNOWLEDGMENTS}

This research was funded by the Ministry of Economy and Competitiveness under project SAFEFRYING (AGL2015-64234-R). The authors thank Ms. R. Alonso, I. Alvarez, Ms. B. Díaz, Ms. S. Jiménez and Mr. MA. Martinez for their technical assistance, and the Spanish Association 'Marcas de Restauración' in the recruitment of the restaurants.

\section{APPENDIX A. SUPPLEMENTARY DATA}

Supplementary data to this article can be found online at https://doi.org//0.1016/j.lwt.2018.10.050.

\section{REFERENCES}

AOAC (1995). Association of official analytical chemists (16th ed.). Washington, DC: Association of Official Analytical Chemists Inc.

Bradshaw, E., \& Ramsay, G. (2017). Potato origin and production. In S. Jaspreet, \& K. Lovedeep (Eds.). Advances in potato chemistry and technology (pp. I-26). San Diego: Academic Press.

Capuano, E., Oliviero, T., Açar, Ö.Ç., Gökmen, V., \& Fogliano, V. (2010). Lipid oxidation promotes acrylamide formation in fat-rich model systems. Food Research International, 43, $1021-1026$.

Dobarganes, M. C., \& Márquez-Ruiz, G. (1998). Regulation of used frying fats and validity of quick tests for discarding the fats. Grasas $Y$ Aceites, 49, 331-335.

EFSA (20I5). Scientific opinion on acrylamide in food. EFSA Journal, 13, 4I04. European Commission (EU) (2017). Commission Regulation 2017/2158 of 20 November 2017 establishing mitigation measures and benchmark levels for the reduction of the presence of acrylamide in food. Official Journal of the European Union, L304, 24-44.

Farkas, B. E., Singh, R. P., \& Rumsey, T. R. (1996). Modeling heat and mass transfer in immersion frying. Part I: Model development. Journal of Food Engineering, 29, 21 I-226.

Fiselier, K., \& Grob, K. (2005). Legal limit for reducing sugars in prefabricates targeting $50 \mu \mathrm{g} / \mathrm{kg}$ acrylamide in French fries. European Food Research and Technology, 220, 45I-458.

Fiselier, K., Hartmann, A., Fiscalini, A., \& Grob, K. (2005). Higher acrylamide contents in French fries prepared from "fresh" prefabricates. European Food Research and Technology, 22I, 376-38I.

FoodDrinkEurope (2013). The acrylamide toolbox, Vol. 57. Food Drink Europe.

Gertz, C., \& Klostermann, S. (2002). Analysis of acrylamide and mechanisms of its formation in deep-fried products. European Journal of Lipid Science and Technology, 104, 762-77I.

Lachat, C., Nago, E., Verstraeten, R., Roberfroid, D., Van Camp, J., \& Kolsteren, P. (2012). Eating out of home and its association with dietary intake: A systematic review of the evidence. Obesity Reviews, 13, 329 -346 .

Medeiros Vinci, R., Mestdagh, F., Van Poucke, C., Kerkaert, B., de Muer, N., Denon, Q., et al. (20II). Implementation of acrylamide mitigation strategies on industrial production of French fries: Challenges and pitfalls. Journal of Agricultural and Food Chemistry, 59, 898-906. 
Mesias, M., Delgado-Andrade, C., Holgado, F., \& Morales, F. J. (2018). Acrylamide content in French fries prepared in households: A pilot study in Spanish homes. Food Chemistry, 260, 44-52.

Mesías, M., Holgado, F., Márquez-Ruiz, G., \& Morales, F. J. (2017). Impact of the characteristics of fresh potatoes available in-retail on exposure to acrylamide: Case study for French fries. Food Control, 73, 14071414.

Mesías, M., \& Morales, F. J. (2015). Acrylamide in commercial potato crisps from Spanish market: Trends from 2004 to 2014 and assessment of the dietary exposure. Food and Chemical Toxicology, 8I, 104-110.

Mestdagh, F., Castelein, P., Van Peteghem, C., \& De Meulenaer, B. (2008). Importance of oil degradation components in the formation of acrylamide in fried foodstuffs. Journal of Agricultural and Food Chemistry, $56,6|4|-6 \mid 44$.

Mestdagh, F., De Meulenaer, B., \& Van Peteghem, C. (2007). Influence of oil degradation on the amounts of acrylamide generated in a model system and in French fries. Food Chemistry, 100, 1153-1159.

Miller, G. L. (1959). Use of dinitrosalicylic acid reagent for determination of reducing sugars. Analytical Chemistry, 31, 426-428.

Palazoglu, T. K., \& Gökmen, V. (2008). Development and experimental validation of a frying model to estimate acrylamide levels in French fries. Journal of Food Science, 73, El09-EII4.

Palermo, M., Gokmen, V., De Meulenaer, B., Ciesarova, Z., Zhang, Y., Pedreschi, F., et al. (2016). Acrylamide mitigation strategies: Critical appraisal of the FoodDrinkEurope toolbox. Food \& Function, 7, 2516-2525.

Pedreschi, F. (2009). Fried and dehydrated potato products. In S. Jaspreet, \& K. Lovedeep (Eds.). Advances in potato chemistry and technology (pp. 319-337). San Diego: Academic Press.

Pedreschi, F., Kaack, K., \& Granby, K. (2006). Acrylamide content and color development in fried potato strips. Food Research International, 39, 40-46.

Pedreschi, F., \& Moyano, P. (2005). Effect of pre-drying on texture and oil uptake of potato chips. LWTFood Science and Technology, 38, 599-604.

Petrie, A., \& Sabin, C. (2009). Medical statistics at a glance (3th ed.). London: Wiley-Blackwell.

Saguy, I., \& Dana, D. (2003). Integrated approach to deep fat frying: Engineering, nutrition, health and consumer aspects. Journal of Food Engineering, 56, 143-I52.

Sanny, M., Luning, P. A., Jinap, S., Bakker, E. J., \& van Boekel, M. A. J. S. (20I3). Effect of frying instructions for food handlers on acrylamide concentration in French fries: An explorative study. Journal of Food Protection, 76, 462-472.

Sanny, M., Luning, P. A., Marcelis, W. J., Jinap, S., \& Van Boekel, M. A. J. S. (2010). Impact of control behaviour on unacceptable variation in acrylamide in French fries. Trends in Food Science \& Technology, 2I, 256-267.

Taubert, D., Harlfinger, S., Henkes, L., Berkels, R., \& Schömig, E. (2004). Influence of processing parameters on acrylamide formation during frying of potatoes. Journal of Agricultural and Food Chemistry, 52, 27352739.

Zamora, R., \& Hidalgo, F. J. (2008). Contribution of lipid oxidation products to acrylamide formation in model systems. Journal of Agricultural and Food Chemistry, 56, 6075-6080.

Zhang, H., Zhang, H., Cheng, L., Wang, L., \& Qian, H. (2015). Influence of deep-frying using various commercial oils on acrylamide formation in French fries. Food Additives \& Contaminants: Part A, I-6. 
FIGURES AND TABLES

Table I. Description of some practices in the preparation of French fries in the different restaurants (R).

\begin{tabular}{|c|c|c|c|c|c|c|c|c|c|c|}
\hline & $\mathbf{R I}$ & $\mathbf{R 2}$ & $\mathbf{R 3}$ & $\mathbf{R 4}$ & $\mathbf{R 5}$ & R6 & R7 & $\mathbf{R 8}$ & R9 & $\mathbf{R I O}$ \\
\hline \multicolumn{11}{|l|}{ potatoes } \\
\hline frozen potato strip & yes & yes & yes & yes & yes & yes & yes & yes & yes & yes \\
\hline supplier selected & yes & yes & yes & yes & yes & yes & yes & yes & yes & yes \\
\hline straight cut & yes & no & yes & yes & yes & no & yes & yes & yes & yes \\
\hline thawing & no & no & no & no & no & no & no & no & no & no \\
\hline RS control & no & no & no & no & no & no & no & no & no & no \\
\hline \multicolumn{11}{|l|}{ Fryer } \\
\hline guidelines for frying & yes & nd & yes & yes & yes & yes & yes & yes & yes & yes \\
\hline frying oil & HOSO & HOSO & HOSO & HOSO & HOSO & HOSO & HOSO & HOSO & HOSO & HOSO \\
\hline heat source & electric & electric & electric & electric & electric & electric & electric & gas & gas & electric \\
\hline Temp. $>175^{\circ} \mathrm{C}$ & no & nd & nd & yes & yes & yes & yes & no & no & yes \\
\hline timer & yes & no & yes & yes & yes & yes & no & yes & yes & yes \\
\hline potato/oil ratio & yes & no & yes & nd & nd & nd & nd & nd & nd & nd \\
\hline potato dedicated & yes & nd & nd & nd & nd & nd & nd & nd & nd & nd \\
\hline dedicated personnel & no & no & yes & no & no & no & yes & no & no & no \\
\hline
\end{tabular}

HOSO (high-oleic sunflower oil), nd (not described), RS (reducing sugars), potatoes (par-fried frozen). 
Table 2. Descriptive analysis of the variables determined in batches of unprocessed and fried potatoes, as well as frying oil provided by the restaurants.

\begin{tabular}{|c|c|c|c|c|c|c|c|c|c|}
\hline & Mean & SD & Min & Max & QI & Median & Q3 & P90 & P95 \\
\hline \multirow{2}{*}{\multicolumn{10}{|c|}{$\begin{array}{l}\text { Unprocessed } \\
\text { potatoes }\end{array}$}} \\
\hline & & & & & & & & & \\
\hline RS (g/kg) & 1.31 & 1.12 & $<\mathrm{LOQ}$ & 4.89 & 0.30 & 1.37 & 2.19 & 2.71 & 3.21 \\
\hline Moisture (\%) & 66.98 & 2.82 & 61.43 & 75.67 & 64.82 & 66.67 & 68.92 & 70.72 & 71.96 \\
\hline \multicolumn{10}{|l|}{ Frying oil } \\
\hline \multicolumn{10}{|l|}{ Polar Compounds } \\
\hline (g/l00 g oil) & 10.44 & 6.84 & 3.30 & 36.80 & 5.80 & 8.50 & 12.50 & 21.03 & 24.38 \\
\hline \multicolumn{10}{|l|}{ Fried potatoes } \\
\hline Acrylamide $(\mu \mathrm{g} / \mathrm{kg})$ & 303 & 196 & $<\mathrm{LOQ}$ & 1068 & 160 & 265 & 386 & 570 & 685 \\
\hline Moisture (\%) & 45.83 & 7.22 & 29.26 & 65.93 & 41.11 & 45.02 & 50.86 & 55.20 & 58.27 \\
\hline$E^{*}$ & 66.79 & 3.79 & 56.11 & 72.85 & 63.74 & 67.62 & 69.76 & 71.07 & 71.49 \\
\hline$L^{*}$ & 63.47 & 3.96 & 53.32 & 70.23 & 60.31 & 63.89 & 66.80 & 68.16 & 68.61 \\
\hline$a^{*}$ & 0.31 & 1.39 & -2.56 & 5.21 & -0.74 & 0.08 & 1.39 & 2.14 & 2.75 \\
\hline$b^{*}$ & 20.48 & 2.54 & 14.36 & 28.11 & 19.19 & $20.4 I$ & 21.94 & 23.72 & 24.73 \\
\hline
\end{tabular}

Reducing sugars (RS). SD: Standard deviation. Min: Minimum. Max: Maximum. QI: First quartile. Q3: Third quartile. P90: $90^{\text {th }}$ percentile. P95: $95^{\text {th }}$ percentile. LOQ: Limit of quantitation. LOQ for RS: $0.2 \mathrm{~g} / \mathrm{kg}$. LOQ for acrylamide: 20 $\mu \mathrm{g} / \mathrm{kg}(\mathrm{n}=\mathrm{III})$. Determinations were done by duplicate for RS, moisture, polar compounds and acrylamide. Four independent measurements were carried out for the colour parameters. 
Table 3. Bivariate correlations between the quantitative variables in frying oil, unprocessed potatoes and French fries, and the formation of acrylamide. Spearman's correlation coefficient $(\rho)$ and level of significance (p-value).

\begin{tabular}{|c|c|c|}
\hline & $\begin{array}{c}\text { Spearman's } \\
\text { correlation }(\rho)\end{array}$ & p-value \\
\hline \multicolumn{3}{|l|}{ Unprocessed potatoes } \\
\hline Reducing sugars $(\mathrm{g} / \mathrm{kg})$ & 0.3586 & $0.0003 * * *$ \\
\hline Moisture (\%) & 0.2238 & $0.0284 *$ \\
\hline \multicolumn{3}{|l|}{ Process } \\
\hline Polar compounds (g/l00 g oil) & -0.0693 & 0.5025 \\
\hline \multicolumn{3}{|l|}{ Fried potatoes } \\
\hline $\mathrm{E}$ & -0.1657 & 0.1067 \\
\hline$L^{*}$ & -0.2117 & $0.0384 *$ \\
\hline$a^{*}$ & 0.7172 & $0.0000 * * * *$ \\
\hline$b^{*}$ & 0.1678 & 0.1021 \\
\hline Moisture (\%) & 0.3832 & $0.0001 * * *$ \\
\hline Moisture loss (\%) & 0.3058 & $0.0024 * *$ \\
\hline
\end{tabular}

Significant values are expressed as $*(p<0.05), * *(p<0.01), * * *(p<0.001), * * * *$ $(p<0.000 I)$ 
Figure I. Box-and-whisker plot and distribution graph for the acrylamide content $(\mu \mathrm{g} / \mathrm{kg})$ in French fries prepared in restaurants $(n=I I I)$. Values are mean $\pm S D$. Dotted line indicates the benchmark level for acrylamide in French fries.

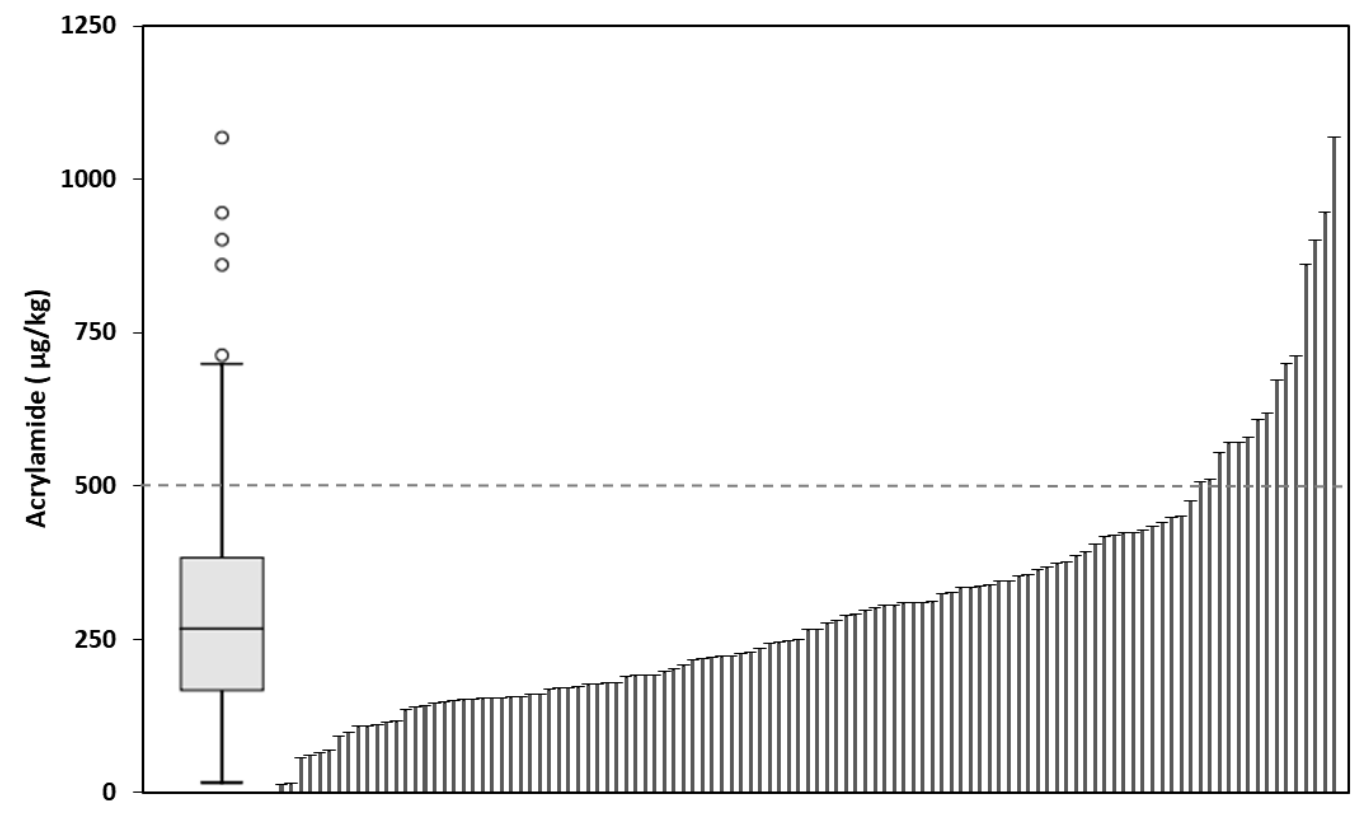


Figure 2. Box-and-whisker plot for (A) acrylamide ( $\mu \mathrm{g} / \mathrm{kg})$ in French fries, and (B) reducing sugars content $(\mathrm{g} / \mathrm{kg})$ in unprocessed potatoes, from ten restaurants. Each restaurant contains data from three different food service establishments. Dots represent outliers.

A

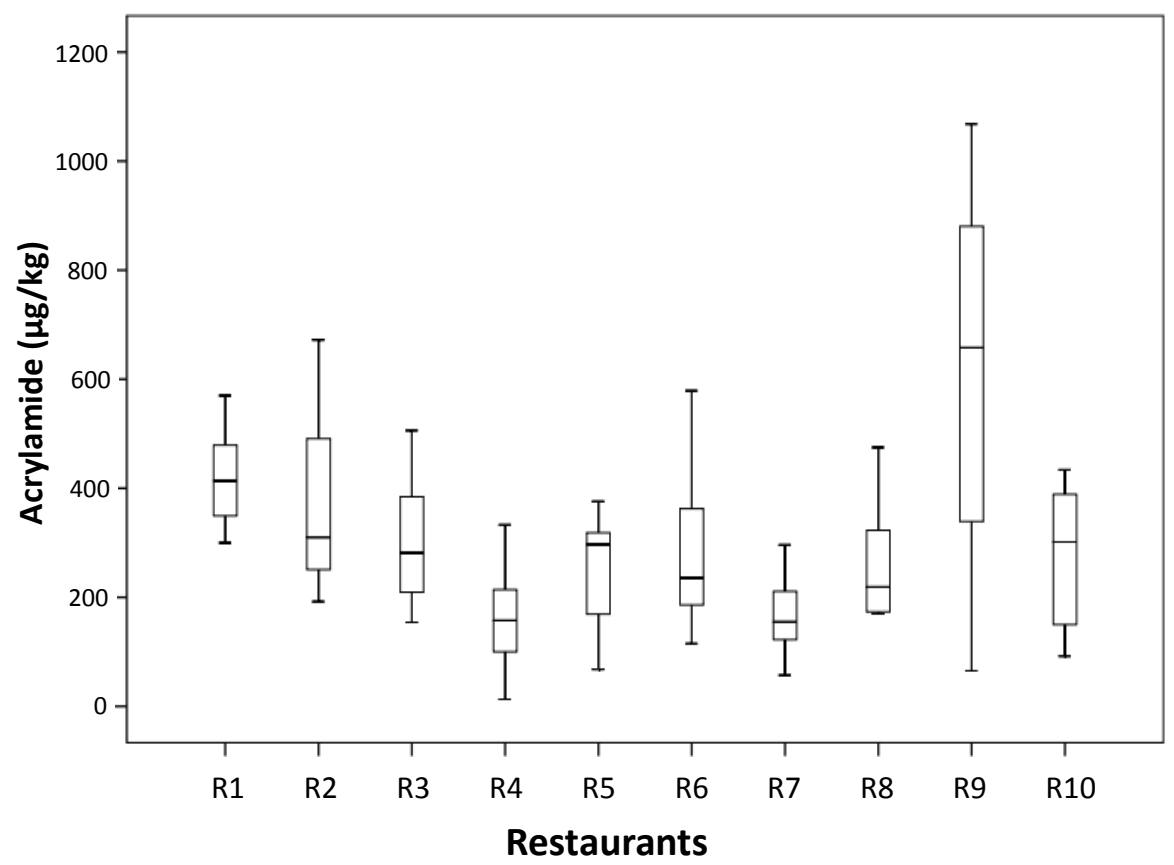

B

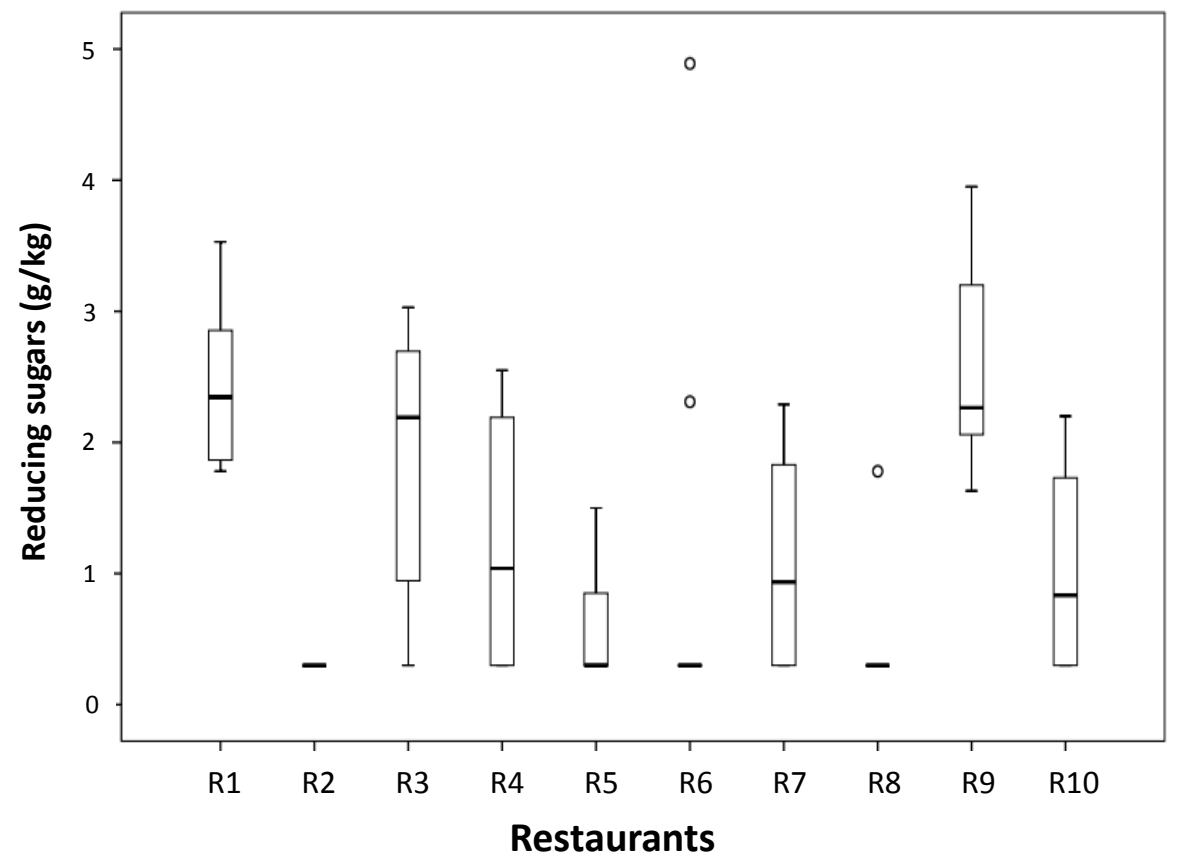


Figure 3. Plot of average acrylamide content $(\mu \mathrm{g} / \mathrm{kg})$ in French fries and reducing sugars content $(\mathrm{mg} / \mathrm{kg})$ in unprocessed potatoes (A); and moisture in the fried product (B). Black circles denote restaurants with rustic chips. White circles denote restaurants with classic French fries straight cut. Dotted line denotes the benchmark level for acrylamide content in French fries. Error bar denotes standard error of the mean.

A

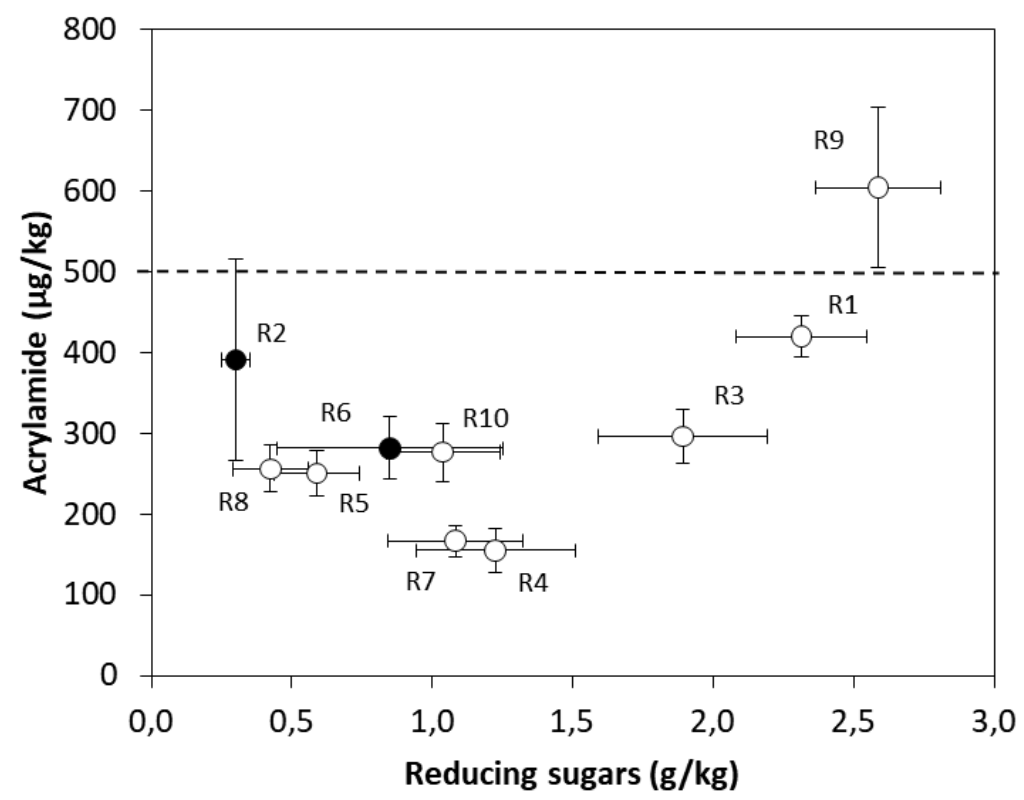

B

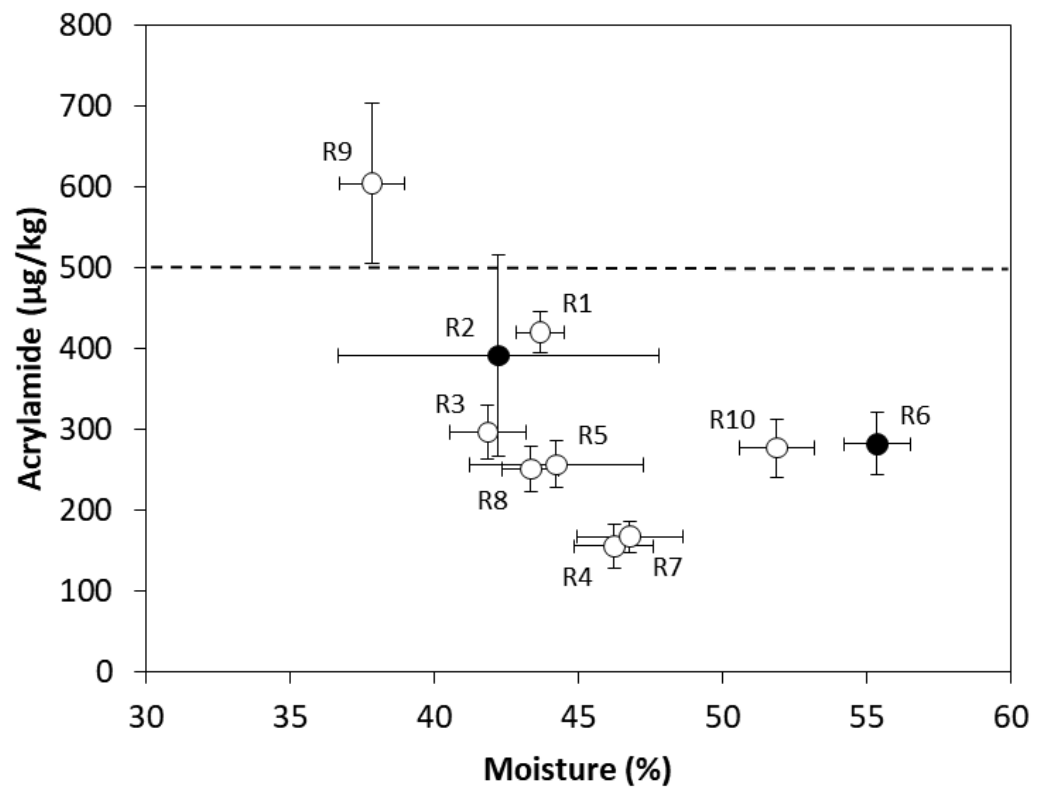


Figure 4. Linear regression between colour parameter $a^{*}$ and acrylamide content $(\mu \mathrm{g} / \mathrm{kg})$ in classical French fries 'straight cut' $(n=96)$ in the restaurants. Dotted lines represent intervals at $95 \%$ of confidence, and solid line the correlation.

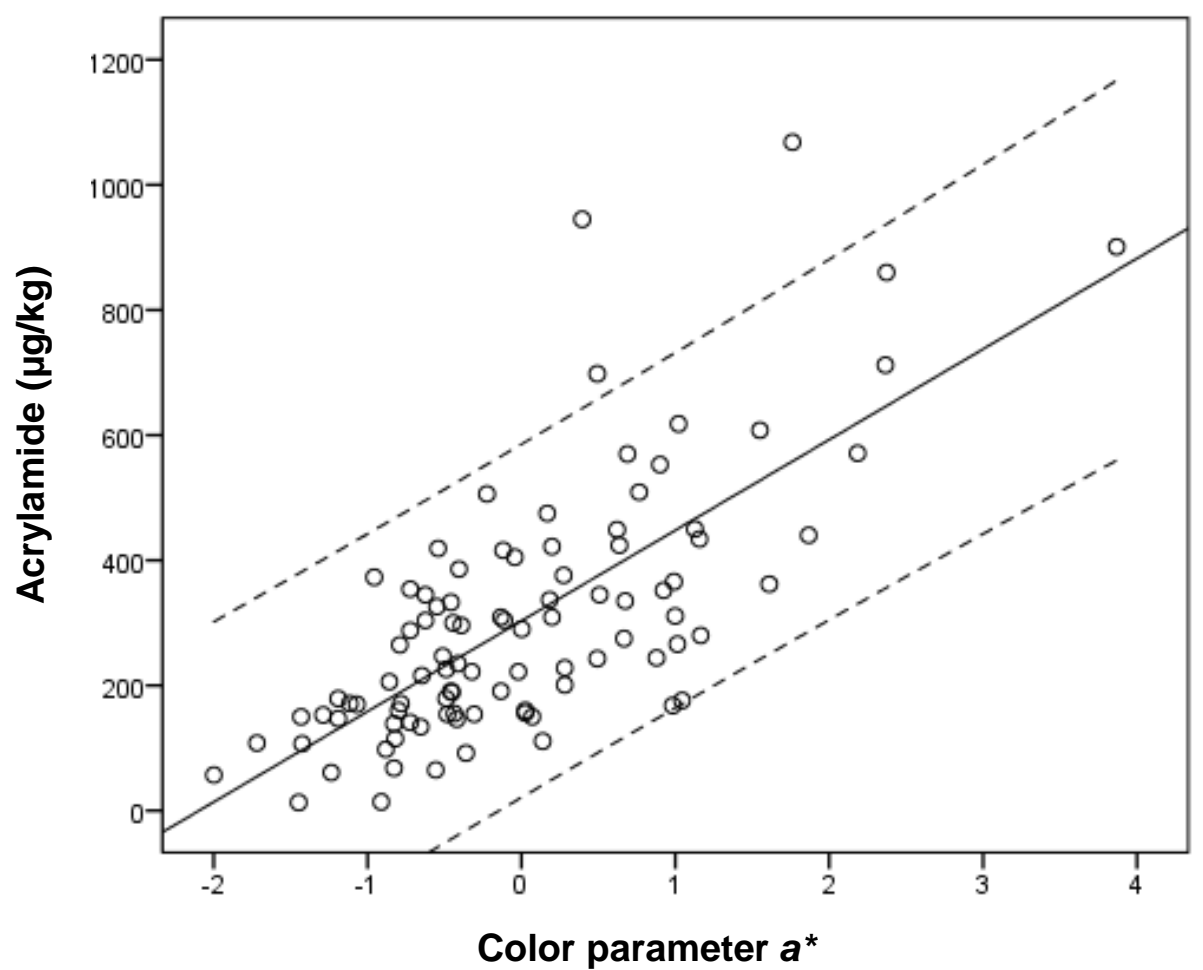


Supplementary material I. Experimental design and sampling for each restaurant $(n=10)$. Samples (unprocessed potato, fried potato, frying oil) were collected at two different moments on the same day (lunch and dinner), for two days in three different establishments from each restaurant (Twelve samples: $2 \times 2 \times 3$ ).

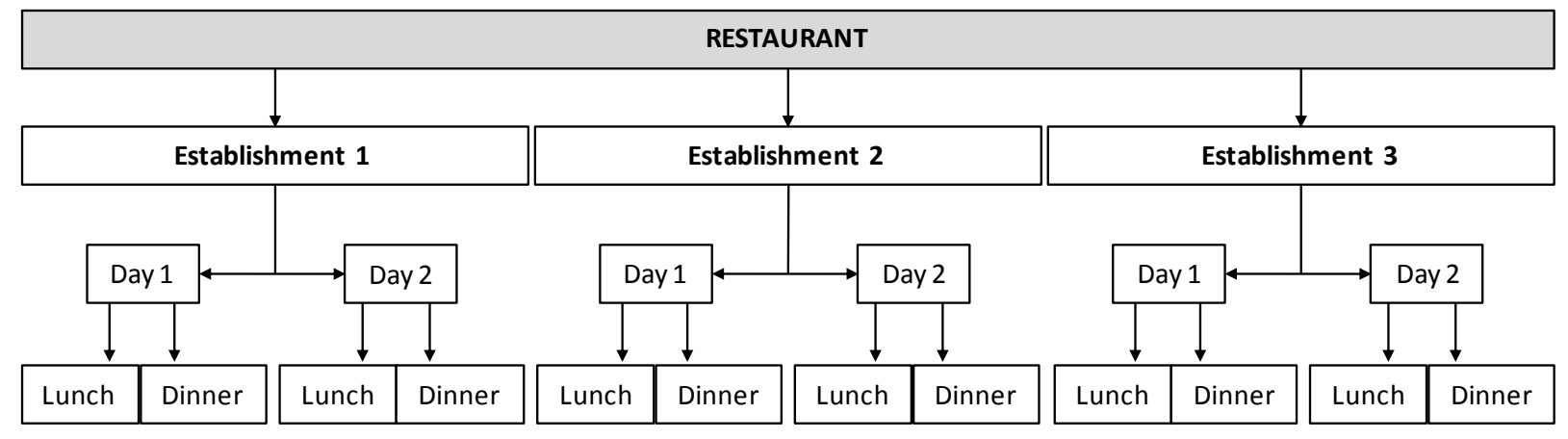


Supplementary material 2. Means plot with $95 \%$ confidence intervals of the acrylamide content $(\mu g / \mathrm{kg})$ in French fries $(A)$ and reducing sugar content $(g / \mathrm{kg})$ in unprocessed potatoes (B) for the different restaurants $(R)$. Black squares denote restaurants with rustic chips. White squares denote restaurants with classic French fries straight cut. Different letter indicate significant differences among restaurants.

A

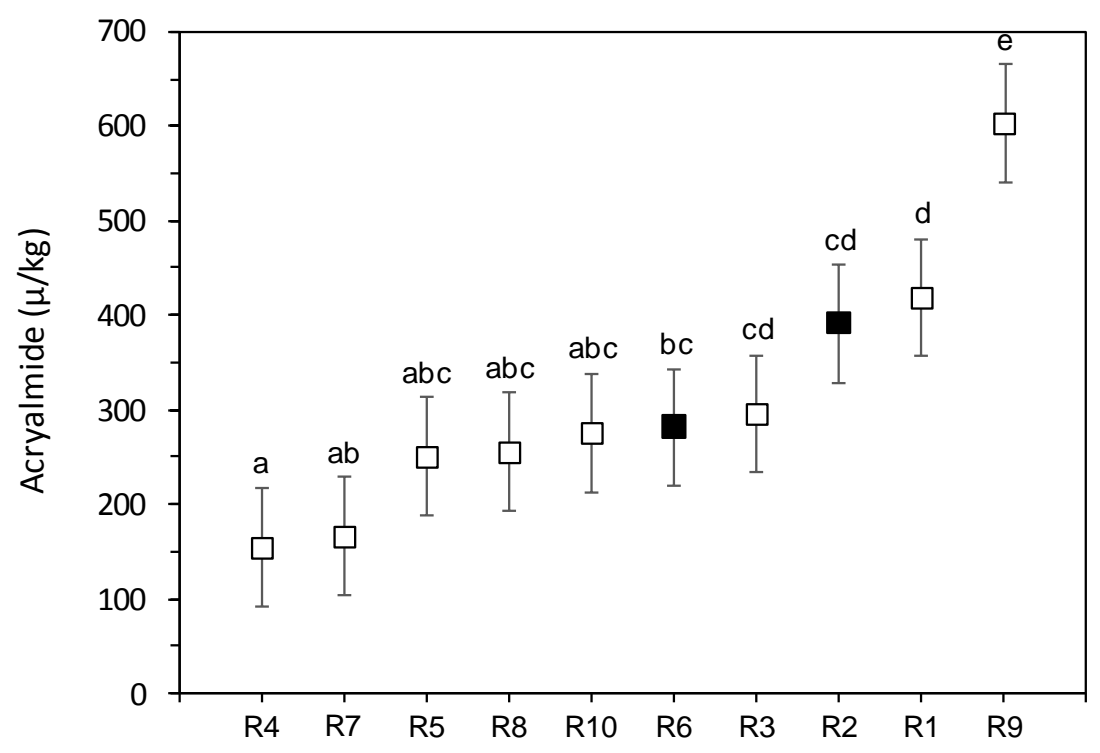

B

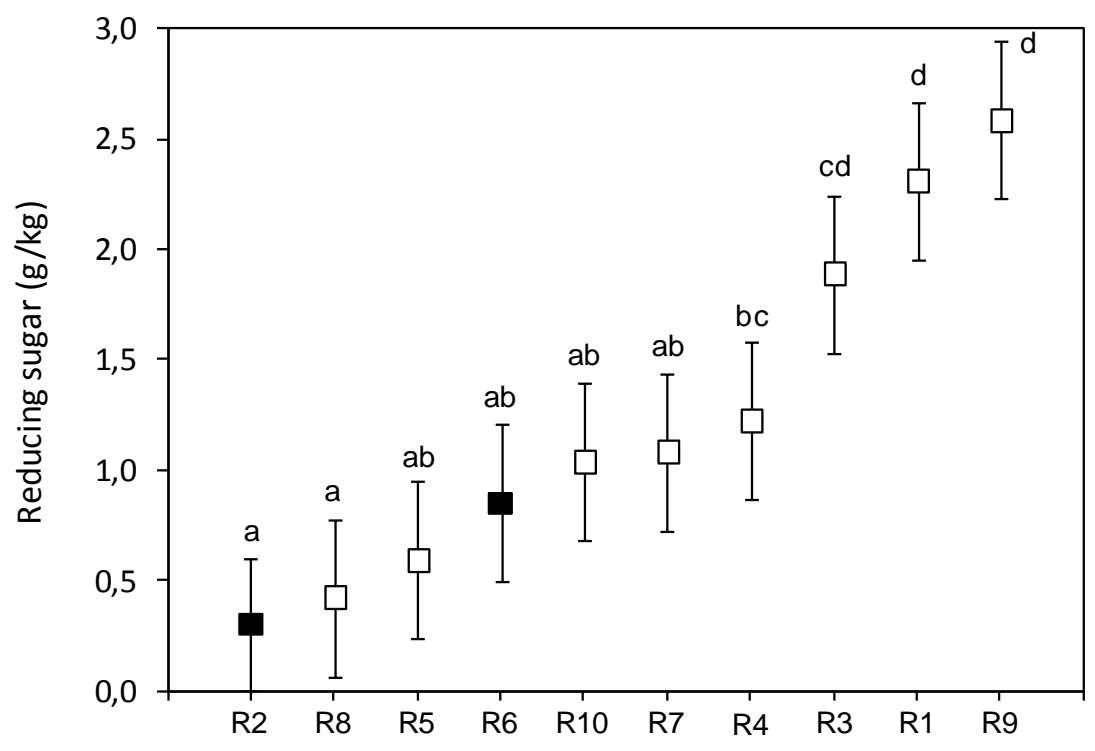

